\title{
Histone Modification Patterns and Their Responses to Environment
}

\author{
Hongzheng Dai $\cdot$ Zhibin Wang
}

Published online: 7 January 2014

(C) Springer International Publishing AG 2014

\begin{abstract}
Histone modifications play important roles in the epigenetic regulation of gene expression. Recent genomewide profiling of histone modifications with deep sequencingbased methods provides novel insights of crosstalk between histone modifications, leading to recent proposals of histone "language" or "web" as an alternative of "code" to appreciate combinatorial modification patterns. Environmental factor-altered histone modifications now may be addressed dynamically and systematically with the recognition and use of these interesting combinatorial patterns.
\end{abstract}

Keywords Histone code $\cdot$ Histone methylation $\cdot$ Histone acetylation $\cdot$ Gene transcription $\cdot$ ChIP-seq $\cdot$ Environmental factors $\cdot$ Histone modification patterns

\section{Introduction}

Histones constitute the core of the nucleosome, the basic structural unit of eukaryotic chromatin. Nucleosome assembly and disassembly are highly dynamic and regulated by incorporation of histone variants and histone modifications $[1,2]$. Posttranslational modifications of histones play important roles not only in shaping the higher-order structure of

\section{H. Dai $\cdot$ Z. Wang}

Laboratory of Human Environmental Epigenomes, Department of Environmental Health Sciences, Bloomberg School of Public Health, Johns Hopkins University, Baltimore, MD, USA

\section{Z. Wang}

The Sidney Kimmel Comprehensive Cancer Center and Department of Oncology, Johns Hopkins University, Baltimore, MD, USA

\section{Z. Wang $(\bowtie)$}

The Epigenetic Center; School of Medicine, Johns Hopkins University, 615 N. Wolfe Street, Baltimore, MD 21205, USA

e-mail: zhwang@jhsph.edu chromatin, but also in modulating transcription regulation, genome stability, and DNA damage repair [2-5]. Various histone modifications have been identified on different components of the histone-nucleus complex [6]. Early on, patterns of posttranslational modifications were discovered at individual amino acid residues on core histones with particular biological functions. Then, at the genome scale, histone modifications were characterized by next-generation sequencing (NGS)-based techniques [7••, 8]. Since then, accumulated data have been able to address the relationships between complicated combinations of histone modifications and their subsequent biological functions $[3,9 \cdot \bullet, 10]$.

It has been noted that epigenetic regulation through histone modification and DNA methylation may be shaped by environmental factors $[11,12]$. A wide range of such environmental factors has been discovered. Chemical pollutants, such as metals and pesticides, may affect histone modifications and/or alter the normal function of enzymes involved in such modifications $[13,14]$. One pathway for metals such as nickel, arsenic, and chromium to affect histone modification is through induced oxidative stress [15]. Dietary factors have been evaluated and have been found to interfere with histone acetylation by inhibiting histone acetyltransferase (HAT)/ deacetylase (HDAC) activities [16, 17]. Environmental factors may affect histone modifications as early as the prenatal stage, when the epigenome may be particularly vulnerable, and cause long-term effects leading to the early development of diseases [18-20]. For long-term exposure and long-term effect, correlations between stress/social environment and histone modifications led researchers to focus on epigenetic regulation of the plasticity of the neurosystem [21].

In this review, we first briefly introduce different types of histone modification and the development of techniques for examining these modifications. We then summarize the characterizations of these histone modifications, especially the characterizations made possible by genome-wide profiling. 
Patterns of particular histone modifications, crosstalk among different modifications, and their possible biological functions are presented. Environmental factors affecting the regulation of posttranslational modifications of histones also are discussed in the context of potential combinatorial patterns of histone modification.

\section{Histone Modifications}

Several chemical groups have been found to bind covalently to each of the four core histones of the nucleosome ( $\mathrm{H} 2 \mathrm{~A}$, $\mathrm{H} 2 \mathrm{~B}, \mathrm{H} 3$, and H4). More than 100 distinct modifications [4] are distributed over 60 residues among histone peptides [6]. Figure 1 shows three types of modification (methylation, acetylation, and phosphorylation) with 39 distinct modifications at 23 residues. Various residue-specific histone modifying enzymes have been identified in the dynamic modification process, whereas modifying enzymes toward to multiple residues have also been reported. Here, we focus mainly on histone acetylation and methylation, two of the most important modifications in terms of both abundance and function.

Histone acetylation is the first histone modification identified 50 years ago by classical physical-chemical analysis [22]. Later, with the help of mass spectrometry, we have learned that most of the acetylated sites are positively charged lysine residues, except for a very few serine residues. For example, in mouse brain, there are 38 lysine sites and only one serine site acetylated on the tails of all four histone proteins [23]. Acetyl groups add negative charges to neutralize positively

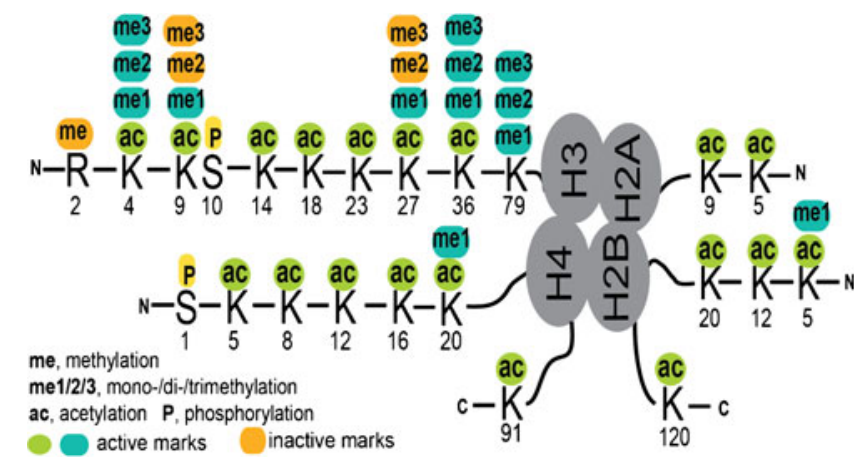

Fig. 1 Different types of histone modifications and their potential crosstalk in regulation of gene transcription. Three major modifications (lysine acetylation, arginine/lysine methylation, and serine phosphorylation) were presented. The 39 distinct modifications at 23 residues on four core histones ( $\mathrm{N}$ or $\mathrm{C}$-terminal tails) were also illustrated. Marks with background color in green or turquoise tend to co-exist in the active genes, whereas marks with background color in orange are likely to coexist in the inactive genes [9••]. Except active marks and inactive marks from the same residue (for example, H3K27ac and H3K27me3), both acetylation marks and active methylation marks can be potentially detected with silent methylation marks (such as $\mathrm{H} 3 \mathrm{~K} 27 \mathrm{me} 3$ ) at the promoter regions of a subset of genes in the human genome [7••]. This subset of genes with the aforementioned 'bivalent marks' are poised for gene induction/silencing charged lysine. Such neutralization might decrease the interaction between histones and DNA, thus making DNA more accessible for transcription. Consistently, unbiased global profiling indicates that all histone acetylation is linked to gene activation (detailed later). Redundancy of acetylated H3 Nterminal lysine residues was revealed by systematic point mutations on $\mathrm{H} 3$ tails in yeast [24]. The number of acetylated lysine residues on histone $\mathrm{H} 3$, rather than their particular positions, is important in affecting transcription activities [24].

Compared to histone acetylation for activation, histone methylation has a more complex role in regulation of gene expression. Unlike the acetyl group, multiple methyl groups may be added to the same amino acid residue to form different methylation statuses (Fig. 1). Lysine may have mono-, di-, and trimethyl groups, whereas arginine may be mono- or dimethylated. Not only do different methyl statuses have different functional outcomes, but methylations at different positions possess different functions. For example, H3K4 trimethylation (H3K4me3) and $\mathrm{H} 3 \mathrm{~K} 36 \mathrm{me} 3$ may be grouped as marks for active genes, whereas $\mathrm{H} 3 \mathrm{~K} 9 \mathrm{me} 3$ and $\mathrm{H} 3 \mathrm{~K} 27 \mathrm{me} 3$ represent repressive marks (Fig. 1). In addition, H3K4me3 may be mainly implicated in transcription initiation, whereas H3K36me3 in elongation. Accordingly, position- and methylation status-specific histone methyltransferase/demethylase have been identified $[25,26]$.

There are other histone modifications with site-specific effects on nucleosome dynamics, gene regulation, or DNA damage response. For example, yeast histone phosphorylation at H3S129 (H3S129ph) may occur within 30 minutes at the adjacent region of double-strand breaks in response to DNA damage, whereas H4S1 phosphorylation also may be induced by genotoxic stresses [27]. On the other hand, H2A/H2B ubiquitination, although occurring at certain lysine sites, does not seem to work in a site-specific manner because of redundancy similar to that of histone acetylation on $\mathrm{H} 3$ [28].

Observations of the variety of histone modifications, their site-specific effects, and the coordinated or antagonistic actions among different modifications lead to a very interesting question: Are there particular histone modification patterns for which we can decipher specific biological meanings in certain contexts? In fact, the original "histone code" hypothesis (see details following) derived from investigations of limited loci suggest such a scenario; that is, certain combinations of histone modifications may be "translated" in a way mimicking the "genetic code" [29]. However, recent global profiling aided by deep sequencing-based methods gradually revealed the whole iceberg. It seems it is more complicated than a simple code. Alternative hypotheses, including "histone web" or "histone language," have been proposed from current large data collections [30-33]. Progress in appreciation of original hypothesis and recent new hypotheses will be detailed later. 


\section{Code/Language in the Context of "-Nomics"}

\section{The Development of Necessary Techniques}

To define the role of a particular histone modification, chromatin immunoprecipitation (ChIP) and ChIP-derived techniques are necessary. Generally, once a particular type of histone modification has been identified, antibodies recognizing such a modification are raised for immunoprecipitation of epitope-associated DNA. ChIP-quantitative PCR (qPCR) was first developed to study patterns of histone modification in a locus-specific way by using targeted PCR primers. ChIP-qPCR still is the standard assay when small sets of interested loci are considered.

Later, hybridization-based chip array was introduced to study the genomic distribution of DNA fragments pulled down by ChIP. This "ChIP-on-chip" or "ChIP-chip" assay provides genome-wide coverage and a fast run time [34, 35]. Although ChIP array has great advantages over ChIP-qPCR, it has its own limitations. Large set numbers are required for better resolution and coverage, signals on repetitive sequences are hard to measure, and system bias may be introduced during amplification [36]. Meanwhile, a Sanger sequencingbased genome-wide approach combining ChIP and serial analysis of gene expression (SAGE) has been developed $[37,38]$. The ChIP-SAGE method was explored further by Zhao's group, finally leading to the revolutionary ChIPsequencing (ChIP-seq) technique.

While the ChIP-chip assay was still maturing and becoming applied more broadly, the transcription/epigenetic field heralded the revolutionary deep sequencing-based method: ChIP followed by massive parallel sequencing (ChIP-seq). The advent of the ChIP-seq method opened a new epigenomic era [39]. As demonstrated by comprehensive characterizations of the human histone methylome and acetylome $[8,9 \bullet \cdot]$, the ChIP-seq method is a cost-effective, high-resolution, reliable technique. Without arbitrary calling of significance from hybridization array signals, ChIP-seq eliminates the risk of introducing bias. Furthermore, ChIP-seq provides digitized data that can be checked visually on the University of California Santa Cruz (UCSC) Genome Browser. Arguably ChIP-seq is the best method available for validating a ChIP assay. Many published ChIP-PCR assays or ChIP-chip results have shown limited success. Such limited success is understandable given that many parameters of ChIP assays affect the final results. For example, corepressor HDACs have been reported in many publications to bind to silent genes [40]. However, the concept of "corepressor-binding silent genes" changed with the demonstration via ChIP-seq analyses of both coactivator HATs and corepressor HDACs binding to active genes in the human genome $[7 \bullet \bullet$. The ChIP-seq method is replacing other methods introduced above to study the genome-wide distribution of histone modifications, transcription factors, and chromatin remodelers.
Human Histone Methylome

The ChIP-seq method brought us the first comprehensive characterizations of histone methylations in the human genome. With antibodies against 20 histone methylations, ChIP-seq analyses reveal their distinct distribution patterns in primary human $\mathrm{CD} 4^{+} \mathrm{T}$ cells. Although not fully understood, the distinct patterns may suggest different functional roles during gene transcription. For example, histone marks of mono, di-, and trimethylation on histone 3 lysine 4 (H3K4me1, -2 , and -3 ) are associated with active genes (Fig. 1). Whereas H3K4me3 signals center around the transcription starting site (TSS), those of H3K4me1 and H3K4me2 spread out toward the gene body or upstream of the TSSs. Other active marks, H3K36me3 and $\mathrm{H} 3 \mathrm{~K} 79 \mathrm{me} / 3$, localized mainly in transcribed regions, also show different distributions: H3K36me3 peaks toward the transcription termination site (TTS) and is associated with transcription elongation, whereas $\mathrm{H} 3 \mathrm{~K} 79$ me $2 / 3$ methylation inclines to the $5^{\prime}$ end of transcribed regions. For methylation status at $\mathrm{H} 3 \mathrm{~K} 9$ and $\mathrm{H} 3 \mathrm{~K} 27$, monomethylation was found to be associated with an actively transcribed region whereas di- and trimethylation work as repressive marks. Moreover, each lysine methylation has its own characteristically enriched genomic regions, distinguished from one another. The aforementioned distribution patterns of selected modifications were illustrated previously in [41] and also summarized in Table 1. Such signature-like distribution has been observed even at nongenic regions [42]. It is worthy to note that although distinct roles of each lysine methylation have been observed with similarity in mammalian embryonic cells [43], the aforementioned methylome patterns are quite different from those in yeast and higher plants. The former has differences in the distribution patterns of H3K4 methylation and H3K36me3 [2, 41]. The latter has no K79 methylation, more K4 dimethylation, and K20 acetylation [44]. One explanation might be their different genomic compositions.

\section{Human Histone Acetylome}

The same approaches have been applied in studying 18 histone acetylations in human primary $\mathrm{CD}^{+}$T cells $[7 \bullet \bullet, 9 \bullet \bullet]$. All histone acetylation modifications are well correlated with active transcription (Fig. 1), which clears the controversial point of whether histone acetylation is involved in gene silencing. These modifications also exhibit different target regions for different acetylation forms. H3K9ac, H3K36ac, and $\mathrm{H} 3 \mathrm{~K} 27 \mathrm{ac}$ have peaks around the TSS region, whereas H4K16ac is enriched at the promoter and transcribed gene body (Table 1). Interestingly, ChIP-seq data suggest that the histone deacetylase HDACs also were enriched at the actively transcribed gene body region $[7 \bullet \bullet]$. This indicates that the process of "writing" and "erasing" of acetyl of histone tails is quite dynamic at these genes in association with both HATs 
Table 1 Summary of histone methylation and acetylation grouped with active/inactive genes*

*Note: Five histone acetylation marks presented in the table can be bivalent marks, if these marks are examined in cells with HDAC inhibitors $[7 \cdot \bullet]$.

\begin{tabular}{lll}
\hline Histone & Pattern of Distribution & Indication of transcription \\
\hline Hodifications & & \\
H3K4me3 & Around TSS & Active gene/bivalent gene \\
H3K4me1 & Around TSS & Active gene/bivalent gene \\
H3K9me1 & Around TSS & Active gene/bivalent gene \\
H3K27me1 & From TSS through gene body & Active gene \\
H4K20me1 & From TSS through gene body & Active gene \\
H2BK5me1 & From TSS through gene body & Active gene \\
H3K79me2/3 & From TSS through gene body & Active gene \\
H3K36me3 & From TSS to the middle of gene body & Active gene \\
H2A.Z & From middle to the end of gene body & Active gene \\
H3K9ac & Around TSS & Active gene/bivalent gene \\
H3K18ac & Around TSS & Active gene \\
H2BK12ac & Around TSS & Active gene \\
H4K12ac & Around TSS & Active gene \\
H4K16ac & From TSS through gene body & Active gene \\
H3K9me2 & From TSS through gene body & Active gene \\
H3K9me3 & From TSS through gene body & Inactive gene/bivalent gene \\
H3K27me2 & From TSS through gene body & Inactive gene/bivalent gene \\
H3K27me2 & From TSS through gene body & Inactive gene/bivalent gene \\
\hline & From TSS through gene body & Inactive gene/bivalent gene \\
\hline
\end{tabular}

and HDACs. Accordingly, ChIP-seq data derived from HDAC inhibitor-treated MC3T3 cells show a genome-wide increase of $\mathrm{H} 4$ acetylation, except $500 \mathrm{bp}$ upstream of the TSS [45].

Not only do different histone acetylation modifications show different distribution patterns steadily, but differential dynamics of acetylation changes at different sites also were observed. For example, 24 hours after the viral oncogene ela transfected into IMR90, $95.4 \%$ of previous H3K18ac peaks diminished, resulting in a two-thirds total reduction of H3K18ac, with new H3K18ac peaks emerging around the promoter region of genes related to cell replication. In contrast, H3K9ac shows a gain of $28 \%$ after transfection [46].

\section{Histone Code/Combinatorial Status/ Bivalent Status in the Genome}

Different histone modification patterns may have distinct biological roles, which prompted the proposal of an influential and dominant "histone code" hypothesis in the field: A particular combination of histone modifications may work as codes that can be read out by specific protein factors, leading to a unique biological outcome. This histone code hypothesis [29] may be used to explain the relationships between histone modifications and their subsequent biological outcomes in a simplified form. Such an analogue to the genetic code inspired researchers trying to find certain simple and elegant relationships. Early investigations on modifications at neighboring residues revealed an antagonizing mode or additive fashion for regulation of gene expression. For example, extensive ChIP-qPCR assays were used to show that H3R2 methylation and $\mathrm{H} 3 \mathrm{~K} 4$ methylation act antagonistically, with the former inhibiting gene expression and the latter stimulating gene expression (Fig. 1) [47]. Although effective in providing insights, this "case-by-case" study of all the potential combinatorial patterns of more than 100 modifications with ChIPqPCR assay is too time consuming to finish in a foreseeable time frame. The introduced human histone methylome and acetylome mentioned earlier provide a chance to extensively determine any potential combinations between two modifications or among multiple modifications. The latter would be a challenge using the traditional ChIP-qPCR method.

In line with modifications for either gene activation or gene silencing, 39 histone marks were examined and may be classified into two groups: modifications in association with silenced genes and those associated with active genes (Fig. 1). Generally speaking, silent marks tend to coexist, as do active marks. For example, silent marks such as H3K27me3 and H3K9me3 both may be associated with silent genes.

Intriguingly, a subset of genes in the human genome is associated with both inactive marks, such as H3K27me3, and active marks, such as $\mathrm{H} 3 \mathrm{~K} 4 \mathrm{me} 3$. It should be pointed out that the active marks can be histone acetylations (Fig. 1) [7••]. These genes are inducible or poised genes bearing these "bivalent" marks. Originally, such "bivalence" was proposed as stem cell specific [48]; however, many differentiated cells 
such as $\mathrm{CD} 4^{+} \mathrm{T}$ cells also have bivalence [49]. Currently, we expect that such bivalency is most likely reflecting an "intermediate" stage during transcription cycles. Indeed, these bivalent genes are transcribed by RNA polymerase II (Pol II) at a short distance before Pol II falls off [7••, 50]. In embryonic stem cells (ESCs), genes with bivalence are most likely to be developmentally regulated genes [51]. Dysregulation of H3K4me3 at bivalent loci in ESCs by depletion of Dpy-30, a core subunit in the SET1/MLL histone methyltransferase complex, does not affect the self-renewal of ESCs but changes its differentiation potential significantly [52]. Interestingly, such dysregulation does not seem to affect the stress response in ESCs.

As described earlier, certain combinatorial patterns seem to be associated with particular biological functions at specific regions, including both TSSs and enhancers. For coexisting silent marks, H3K27me3 seems to have a dominant role, because genes with this mark most likely are silenced (Fig. 2) [9••]. The aforementioned observation also applies to bivalent genes. Though in association with both active marks including $\mathrm{H} 3 \mathrm{~K} 4 \mathrm{me} 3$ and silent mark $\mathrm{H} 3 \mathrm{~K} 27 \mathrm{me} 3$, these bivalent genes are not associated with full-length RNA transcripts rather than with short RNA molecules (up to 65 nucleotides) [50]. It is expected that inactive histone marks (H3K9me3 and/or H3K27me3)-resulted compact chromatin structure contributes to the falling off of PolII.

Compared with a less complex combination of silent marks for silent genes, active genes may be associated with many more active marks and thus have a very complex combination of patterns [9*0]. Theoretically, any two or multiple active marks can form a combinatorial pattern or "code" and the number of potential combination out of 39 marks is huge. In reality, however, limited combinations (little over 4000 combinatorial patterns) are detected $[9 \bullet \bullet]$. Limited combinatorial patterns suggest the co-existence of multiple active marks. Indeed, a common module (or backbone) consisting of 17 modifications across more than 3000 promoters exists in the human genome. The 17 modifications include H2BK5ac, H2BK12ac, H2BK20ac, H2BK120ac, H2A.Z, H3K4ac, H3K4me1, H3K4me2, H3K4me3, H3K9ac, H3K9me1, H3K18ac, H3K27ac, H3K36ac, H4K5ac, H4K8ac, and $\mathrm{H} 4 \mathrm{~K} 91 \mathrm{ac}$, presenting in 821 different patterns. Adding more active marks to this module, associated genes seem to have increased expression [9••] (Fig. 2). The existence of this module suggests that multiple active marks, largely histone acetylation marks, work in a concerted fashion to robustly facilitate gene expression upon external signal stimulation.

Notably, there is no specific combination of two or more marks that determines the expression of one gene or a group of genes. Genes bearing the same combinatorial pattern composed of multiple marks actually have diversified expression, from low to extremely high (Fig. 2, with permission from the original publisher $[9 \cdot \bullet])$. It seems that the solely combinatorial pattern of histone modifications cannot specify a unique biological outcome, transcription level of genes. Presumably, additional players including transcription factors and DNA methylation may contribute to the specification of outcomes. However, it should be pointed out that the magnitude of each histone mark in our studies was not taken into consideration when analyzing the combinatorial patterns, partially because of the related complexity. For example, quantitative analyses require some sort of normalization of ChIP-seq signals from different antibody-pulled DNA. Readers are alerted that such quantitative analyses are expected to more accurately correlate combinatorial patterns with gene expression, because it is well known that the magnitude of active histone modification signals is positively correlated with gene expression $[9 \bullet \bullet, 53]$.

On the other hand, there are intrinsic unsolved problems in this code hypothesis $[30,54]$. There are no strict "one-to-one" or "multiple-to-one" relationships that can be applied dominantly among various combinatorial patterns of histone modification [10]. The lack of dominant effects for point mutations at critical histone residues affecting global or tissue-specific biological functions further undermine the validity of such a
Fig. 2 Genes bearing the same combinatorial pattern could have different expression levels. B stands for "Backbone" of 17 modifications. Numbers in each combinatorial pattern indicate how many genes are categorized in this group. (Modified with permission from: Wang Z, Zang C, Rosenfeld JA, Schones DE, Barski A, Cuddapah S, Cui K, Roh TY, Peng $\mathrm{W}$, Zhang MQ, et al. Combinatorial patterns of histone acetylations and methylations in the human genome. Nat Genet 2008, 40:897-903) [9••]

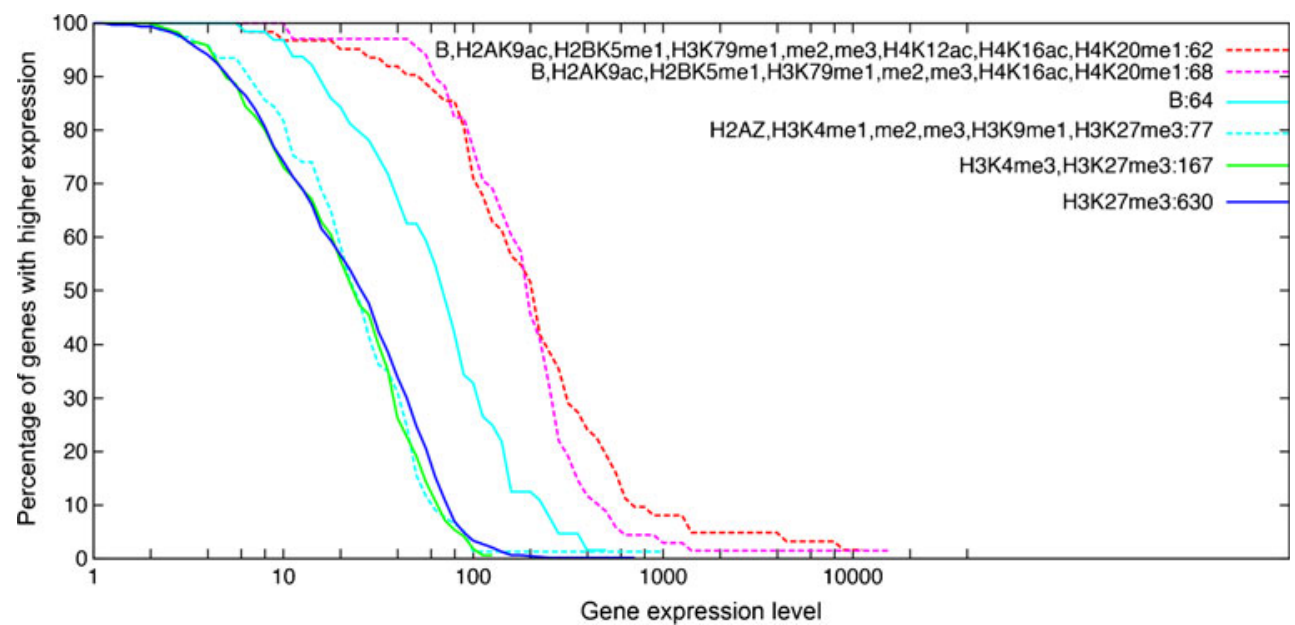


simple code model $[55,56]$. Moreover, the highly dynamic process of nucleosome reconstitution makes a steady code reading very difficult to regulate various biological functions [54]. Therefore, more nonhistone components/effectors would be required to incorporate into bigger module complexes. Such complexes may work in a far more complicated way than a simple code could explain [30,54]. Stepwise processes or web-like interactions are possible alternative scenarios $[3$, 30]. Complicated crosstalk revealed among H3K9ac, H4K16ac, and H3K4 methylation in human T cells [7••] prompted Lee and colleagues [33] to propose a language of histone modifications.

\section{Environmental Factors Affect Histone Modification Patterns}

Diseases caused by environmental factors have drawn a great deal of attention from clinicians and researchers for decades. Heritable DNA mutations caused by radiation and chemical agents have been well documented. However, only recently was it realized that environment largely can shape the transcriptome/metabolome to cause diseases epigenetically, i.e., beyond the interaction to change the DNA sequence directly $[11,20]$. Environmental epigenetics has focused on possibly heritable alterations in DNA methylation and/or histone modifications through environmental influences and how those changes might be related to the variability in risk/severity of diseases modifiable by the environment [20]. Limited by the availability of samples from subjects, most environmental epigeneticists work on DNA methylation, especially those investigating prenatal exposures causing epigenetic changes in the fetus [57]. Recent progress in the ChIPseq technique using a low cell number and fixed pathologic tissue largely increased the dataset of histone modifications influenced by environmental factors [58]. Here, we review some examples of histone modification changes affected by some environmental factors.

A. Specific examples of exposures and effects on histone modifications

\section{Arsenic}

Neurologic disorders and a higher risk of carcinogenesis caused by chronic exposure to arsenic in drinking water have long been a global public health concern. Arsenic may affect DNA methylation and histone methylation primarily by competing with the methyl group S-adenosylmethionine (SAM) during its own methylation metabolism [15]. A159 cells treated with arsenite had increased $\mathrm{H} 3 \mathrm{~K} 4 \mathrm{mel}$ and $\mathrm{H} 3 \mathrm{~K} 4 \mathrm{me}$, whereas the H3K4me3 level decreased [59]. Not only were distinct dynamics observed in histone methylation patterns affected by arsenic, but crosstalk between different histone modifications also was reported. For example, global changes of H4K16ac were observed by mass spectrometry assay in human bladder epithelial cells [60]. Most strikingly, recent ChIP-seq data revealed that prenatal exposure to arsenic caused global reduction of $\mathrm{H} 3 \mathrm{~K} 9 \mathrm{ac}$ in mouse offspring [61•]. On the other side, elevation of histone acetylation at the promoter region of certain genes related to those up-regulated by arsenic exposure has been reported [62].

Nickel

Nickel has been considered a carcinogenic agent that is not involved in DNA mutagenesis directly but that damages the inactive heterochromatin region selectively. An earlier report indicated that nickel compounds might induce DNA methylation first, then chromatin condensation to inhibit gene expression [63]. Further investigation discovered that nickel can inhibit H3K9 demethylase selectively by replacing iron from the catalytic center [64]. Western blot analysis from in vitro experiments performed on A549 cells suggests that global H3K4me3 levels increased after 24 hours of $\mathrm{NiCl} 2$ treatment [59]. Accordingly, examination of human subjects exposed occupationally to nickel revealed that the global H3K4me3 level is elevated in the peripheral blood mononuclear cells whereas the H3K9me2 level is reduced [65]. Nickel also may cause global histone hypoacetylation in human hepatoma cells by inhibiting HAT [66], whereas it may induce ubiquitination by inhibiting deubiquitinase in A549 cells [67]. These multiple roles played by nickel in the regulation of epigenetic layers indeed indicate the complexity of histone modification responses to environmental factors.

B. Tissue- and site-specificity of responses in histone modifications to environmental factors

The advantages of ChIP-seq or ChIP-seq-derived techniques enable researchers to look for specific local patterns in addition to the genome-wide changes of histone modification. For example, because nickel can induce global elevation of $\mathrm{H} 3 \mathrm{~K} 4 \mathrm{me} 3$, detailed ChIP-seq data combined with RNA-seq results indicated that nickel exposure not only increases the level but also broadens the range of $\mathrm{H} 3 \mathrm{~K} 4 \mathrm{me} 3$ within $5 \mathrm{~kb}$ of the TSS of nickelinducible genes [68 $]$. In the brains of mouse pups prenatally exposed to arsenic, it was observed that in addition to global hypoacetylation at H3K9, ChIP-seq data suggested enriched representation at the promoter regions of certain types of zinc finger transcription factors, compared with the control group [61•]. 
Global changes of histone modification in response to environmental factors may be tissue- and site-specific. In a study of the asymmetric arginine methylation of histone in rats fed with a methionine-enriched but vitamin Bdeficient diet [69], the level of H3R8me2a in the brain of treated rats was significantly lower than in controls, whereas the level in the liver was unaffected. Two other arginine sites, H4R3 and H3R17, showed no difference in asymmetric methylation between the control and treatment groups for all tissues. Different dynamic profiles of histone modification changes upon osteogenic induction have been reported in two different odontogenic neural crest-derived intermediate progenitor cells [70]. Mineralization-inductive conditions resulted in more pronounced, highly dynamic changes in $\mathrm{H} 3 \mathrm{~K} 4 \mathrm{me} 3$, $\mathrm{H} 3 \mathrm{~K} 9 \mathrm{me} 3$, and $\mathrm{H} 3 \mathrm{~K} 27 \mathrm{me} 3$ at the promoter regions of osteogenic-related genes in dental follicle progenitor cells, whereas those histone marks remained closer to the baseline level in dental pulp progenitor cells.

C. Effect of environment on histone modifications during differentiation

Certain histone modification patterns may emerge during differentiation or even during evolution as an adaptation to the environment. During the adipogenesis of mouse mesenchymal stem cells, although the histone modifications were globally stable, gene-specific distinct and highly dynamic histone modification patterns were observed with a standard ChIP-qPCR assay [71]. In yeast, Zou and colleagues [72] extensively compared genome-wide histone modification patterns with ChIP-on-chip data between duplicated gene pairs. They found there indeed are more conserved histone modification patterns in duplicated gene pairs than in randomly selected counterpart pairs. Further, it is suggested that the difference in such patterns between duplicated gene pairs is higher at the promoter than the open reading frame region. With a recent large set of ChIP-seq data on humans and mice, similar approaches may be used to identify species-specific or conserved histone modification patterns in response to environmental factors. In plants, ChIP-qPCR results in H3K9ac showed similar dynamic patterns in maize and sorghum in response to light at the promoter regions of genes related to C4 metabolism despite no detectable homology at the DNA level [73].

D. Histone changes in short-term response vs. long-term response

Highly dynamic changes in protein synthesis and gene expression are critical for organisms to respond promptly to deleterious environmental factors. Because posttranslational regulation at the protein level affects RNA transcription, histone modifications may play important roles in immediate response pathways dynamically. It has been reported that in HCT116 cells, levels of H3 and H4 acetylation increase at least twofold within 30 minutes after tetradecanoylphorbol acetate (TPA) induction at the upstream region of immediately early gene Fosl1 [74]. On the other hand, 30-minute pretreatment with HDAC inhibitor effectively reduces Fosll expression level upon TPA induction, suggesting an up-regulation role for deacetylation in Fosl1 expression. This case demonstrates that not only is acetylation of $\mathrm{H} 3 / \mathrm{H} 4$ promptly responsive, but both acetylation and deacetylation coordinate to induce the expression of Fosl1, similar to the scenario proposed from comprehensive genome-wide analysis $[7 \bullet \bullet]$.

DNA damage response is critical for cells to survive after ionizing radiation. Within 15 minutes after gamma irradiation of mouse embryonic fibroblast (MEF) cells, $\mathrm{H} 3 \mathrm{~K} 9 \mathrm{me} 2$ and $\mathrm{H} 3 \mathrm{~K} 9 \mathrm{me} 3$ levels are reduced significantly [75]. The KMD4 subfamily of histone demethylase can be recruited quickly (within 100-150 seconds) into DNA damage induced by gamma radiation. Moreover, overexpression of GFP-KMD4 fusion protein can efficiently enhance the DNA damage response by reducing the H3K9 methylation barrier [75]. In mice exposed to gamma radiation, $\mathrm{H} 4$ acetylation levels of cells extracted from bone marrow were reduced by $97 \%$ vs. controls after 4 hours of radiation [76]. Feeding mice with the HDAC inhibitor phenylbutyrate (PB) 24 hours before radiation increased $\mathrm{H} 4$ acetylation levels by $67 \%$ vs. controls and lower than in the PB-only group (207\%). Meanwhile, PB use may reduce about $50 \%$ of the cell apoptosis caused by radiation [76]. This DNA damage protection mediated by HDAC inhibitors may be very useful in cancer radiotherapy [77, 78]. Early histone modifications related to behavior patterns were reported in ventromedial hypothalamus tissue of female mice administered estradiol [79]. In this case, distinct temporal patterns of H3S10phK14ac, H3ac, $\mathrm{H} 3 \mathrm{~K} 4 \mathrm{me} 3$, and $\mathrm{H} 3 \mathrm{~K} 9 \mathrm{me} 3$ rapidly and transiently emerged within 6 hours after estradiol administration. Acute response of histone modifications might be dose dependent when cells are exposed to toxic environmental chemicals. For example, a significant reduction of H4K16ac was reported in human bladder epithelial cells treated with $3 \mu \mathrm{M} \mathrm{As}{ }^{\mathrm{III}}$ after 24 hours, whereas it took 7 days to observe such a reduction with $0.3 \mu \mathrm{M}$ and $1.0 \mu \mathrm{M}$ As ${ }^{\text {III }}$ [60]. Differences in histone response to short-term and long-term exposure also were reported in a cell model testing reactivation of latent HIV virus. Long-term treatment with HDAC inhibitor reactivates the virus in resting $\mathrm{T}$ cells successfully, whereas short-term treatment has a much more limited capability [80].

Long-term exposure to low-level toxic environmental agents, in addition to exposure from acute and high-dosage pollutants, also affects public health. Histone modifications in response to long-term exposure or secondary 
effects on such environmental stimuli exhibit interesting patterns. Seven days of exposure to arsenic at a low level $(1 \mu \mathrm{M})$ increases the global level of H3K4me3 in A549 significantly. This histone modification remains at an elevated level even after 7 days of proliferation, after metals have been removed from the medium [59]. Similar "inheritable" epigenetic marks were reported and called "longterm somatic memory" in plants that underwent transient mild salt treatment followed by extensive growth in control conditions [81]. Pretreated plants showed no growth/ survival advantages over the control group until secondary stress was applied for 2 weeks. ChIP-seq data of the active marks $\mathrm{H} 3 \mathrm{~K} 4 \mathrm{me} 2$ and $\mathrm{H} 3 \mathrm{~K} 4 \mathrm{me} 3$ and repressive marks H3K9me2 and H3K27me3 revealed a tissue-specific, sustained histone modification pattern preferably enriched in transcription factor genes after 24 hours of salt treatment [81]. In human ESC cells continuously treated with retinoic acid, the biggest fold changes in $\mathrm{H} 3 \mathrm{~K} 4 \mathrm{me} 3$ (upregulated) and $\mathrm{H} 3 \mathrm{~K} 27 \mathrm{me} 3$ (down-regulated) levels occurred at day 6 , followed by less change at day 12 , compared with control cells [82]. Therefore, certain time windows may be important to establish the so-called histone memory. In a study of blasticidin S-resistant malaria lines, it was revealed that 4-day treatment with this antimalarial drug apparently sterilized the parasite population, although some mutant lines recovered 4 weeks after blasticidin S was withdrawn [83]. Detailed examination indicated that there was no change at the DNA level in those epimutant lines. In contrast, histone modifications including $\mathrm{H} 3 \mathrm{~K} 9 \mathrm{ac}, \mathrm{H} 3 \mathrm{~K} 9 \mathrm{me}$, and $\mathrm{H} 3 \mathrm{~K} 4 \mathrm{me} 3$ were found to be increased or decreased significantly at the promoter regions of resistance-related genes [83]. Long-term exposure also would make particular histone modification patterns adapt to the existence of such stimuli. For example, mouse model have been used to study the dependency of histone modifications on chronically administrated morphine (9 days) in brain. Within 1 hour after the last administration of morphine, morphine was in vivo precipitated by naloxone. Such precipitation results in different dynamics of $\mathrm{H} 3$ phosphorylation and acetylation: $\mathrm{H} 3$ phosphorylation was increased significantly while $\mathrm{H} 3$ acetylation remained unchanged [84].

Chromatin modifications in response to chronic environmental stimuli, such as the natural light cycle, have long been a hot topic to many geneticists and epigeneticists. Concept of the circadian clock model has been developed based on the study of chronically changed transcriptome/epigenome [85, 86]. Particular histone modifications may play different roles in the establishment and maintenance of such a circadian clock. In Arabidopsis, it was found that although both $\mathrm{H} 3$ acetylation and $\mathrm{H} 3 \mathrm{~K} 4 \mathrm{me} 2 / \mathrm{me} 3$ are essential to the activation of oscillator genes, blocking $\mathrm{H} 3 \mathrm{~K} 4$ trimethylation increases the binding for clock-repressor as a transitional mark [87]. Rhythmic oscillation of $\mathrm{H} 3$ acetylation and $\mathrm{H} 3 \mathrm{~K} 4 \mathrm{me} 2 / \mathrm{me} 3$ is reported to be positively related to rhythmic transcriptions of light/dark cycle-related genes, whereas $\mathrm{H} 3 \mathrm{~K} 36 \mathrm{me} 2$ has a negative correlation [88]. Circadian rhythmic oscillation of histone modifications also were observed in mammals [32, 89, 90]. Using ChIP-seq and the expression tiling array, exact opposite rhythmic oscillations of such antagonistic marks (H3K4me3 and H3K9me3) have been found, with good correlation with the rhythmic transcriptions of circadian clock-related genes. Interestingly, H3K4me3, as opposed to its important role in regulating clock-repressor binding in plants, has no obvious correlation with the number of peak-transcribed genes at a particular time point during the light/dark cycle [91•].

As discussed earlier, histone modifications, whether responding to short-term, long-term, or circadian-type environmental stimuli, may form particular inheritable patterns, usually called "epigenetic memory." Most epigenetic memory studies focus on DNA methylation, which is more stable and more easily detected in transgeneration/ cell cycles. For example, genome-wide bisulfitesequencing data from various mouse tissues suggest that DNA methylation may play a role as a memory marker at the embryonic stage for future differentiation [92]. A study of yeast transcription discovered a particular type of epigenetic memory: "epigenetic transcription memory." In yeast, recently transcribed genes induced by salt stress can be transcribed easily and more abundantly four to five generations later upon oxidative stress because of the formation of a nuclear pore complex (NPC)-transcription machinery complex [93]. A similar phenomenon was reported in human cells in response to interferon- $\gamma$. Those memory-capable genes have a persistent H3K4 dimethylation signal at their promoter region, and this signal is very essential for recruiting Pol II and promoting the formation of the NPC-transcription complex [94].

\section{Conclusions}

The progress of NGS-based genome-wide mapping of histone modifications (e.g., ChIP-seq) provides great opportunities to understand the histone epigenetics responding to environmental stimuli systematically and dynamically [95]. Future investigations might benefit from recently developed techniques, such as targeted NGS in large-scale screening and the combination of ChIP and mass spectrometry, to determine the interactome of histone modification writers and readers [96]. Highly sensitive quantitative mass spectrometry may be used as a sensitive and alternative measure, if the dynamics of histone 
changes must be focused and the amount of available ChIP DNA is limited [97].

As our understanding of histone modification patterns continues to expand, it is becoming possible for researchers to make those codes/languages/patterns practical by modifying the histone landscape in certain contexts. The approval of HDAC inhibitors as anticancer drugs is a successful but simple example of clinical application [78], which targets only the general histone acetylation pattern. Efforts also have been made to manipulate the histone methylome by inhibiting lysine-specific demethylase 1 (LSD1) for $\gamma$-hemoglobin induction [98•]. In this case, the locus-specific $\mathrm{H} 3 \mathrm{~K} 4$ dimethylation pattern is the major target. In the future, combinatorial modulation of histone marks might be considered for more complicated targeting.

The most difficult aspect of understanding histone patterns is that there are multiple layers engaging in crosstalk with one another: histone acetylation, histone methylation, and phosphorylation. One modification affects another kind of modification, thus effecting particular patterns [33]. Making it more complex, crosstalk between histone modifications and another epigenetic modification, DNA methylation, has been reported frequently [99]. Thus, more systematic and comprehensive models including as many epigenetic layers as possible should be in the scope of researchers for future investigations.

Acknowledgments As a Kimmel Scholar from the Sidney Kimmel Foundation for Cancer Research and Lenfant Biomedical Scholar from National Heart, Lung and Blood Institute, U.S.NIH, ZW thanks their support. The work in the Wang laboratory was also supported by grants from US Department of Agriculture, National Institute of Food and Agriculture, Agriculture and Food Research Initiative grant; a HoChing Yang Memory Faculty Award from the Bloomberg School of Public Health; and a startup from the Environmental Health Sciences department. We apologize to the investigators whose important contributions cannot be cited because of space limitations.

\section{Compliance with Ethics Guidelines}

Conflict of Interest Hongzheng Dail and Zhibin Wang declare that they have no conflict of interest.

Human and Animal Rights and Informed Consent This article does not contain any studies with human or animal subjects performed by any of the authors.

\section{References}

Papers of particular interest, published recently, have been highlighted as:

- Of importance

-. Of major importance

1. Fraser P, Bickmore W. Nuclear organization of the genome and the potential for gene regulation. Nature. 2007;447:413-7.
2. Li B, Carey M, Workman JL. The role of chromatin during transcription. Cell. 2007;128:707-19.

3. Suganuma T, Workman JL. Signals and combinatorial functions of histone modifications. Annu Rev Biochem. 2011;80:473-99.

4. Zentner GE, Henikoff S. Regulation of nucleosome dynamics by histone modifications. Nat Struct Mol Biol. 2013;20:259-66.

5. Berger SL. The complex language of chromatin regulation during transcription. Nature. 2007;447:407-12.

6. Kouzarides T. Chromatin modifications and their function. Cell. 2007;128:693-705.

7.• Wang Z, Zang C, Cui K, Schones DE, Barski A, Peng W, et al. Genome-wide mapping of HATs and HDACs reveals distinct functions in active and inactive genes. Cell. 2009;138:1019-31. This paper not only reveals coordinated dynamic processes by HATs and HDACs in modulating combinatorial acetylation patterns of active genes for the first time, but also demonstrates crosstalk between different histone modifications, prompting the proposal by Lee et al. [33] of a language of histone crosstalk.

8. Barski A, Cuddapah S, Cui K, Roh T-Y, Schones DE, Wang Z, et al. High-resolution profiling of histone methylations in the human genome. Cell. 2007;129:823-37.

9.• Wang Z, Zang C, Rosenfeld JA, Schones DE, Barski A, Cuddapah S, et al. Combinatorial patterns of histone acetylations and methylations in the human genome. Nat Genet. 2008;40:897-903. Comprehensive characterizations lead to the first glimpse of what kind of histone code exists in the human genome. Numerous combinatorial patterns are revealed, but none determines the expression level of associated genes. The concept of combinatorial patterns has triggered reconsideration of the simple histone code hypothesis.

10. Rando OJ. Combinatorial complexity in chromatin structure and function: revisiting the histone code. Curr Opin Genet Dev. 2012;22:148-55.

11. Baccarelli A, Bollati V. Epigenetics and environmental chemicals. Curr Opin Pediatr. 2009;21:243-51.

12. Bollati V, Baccarelli A. Environmental epigenetics. Heredity. 2010;105:105-12.

13. Chervona Y, Arita A, Costa M. Carcinogenic metals and the epigenome: understanding the effect of nickel, arsenic, and chromium. Metallomics. 2012;4:619-27.

14. Collotta M, Bertazzi PA, Bollati V. Epigenetics and pesticides. Toxicology. 2013;307:35-41.

15. Chervona $\mathrm{Y}$, Costa $\mathrm{M}$. The control of histone methylation and gene expression by oxidative stress, hypoxia, and metals. Free Radic Biol Med. 2012;53:1041-7.

16. Mathers JC, Strathdee G, Relton CL. 1 - Induction of epigenetic alterations by dietary and other environmental factors. In: Zdenko $\mathrm{H}$, Toshikazu U, editors. Advances in genetics. Academic Press; 2010. p. 3-39.

17. Delage B, Dashwood RH. Dietary manipulation of histone structure and function. Annu Rev Nutr. 2008;28:347-66.

18. Perera F, Herbstman J. Prenatal environmental exposures, epigenetics, and disease. Reprod Toxicol. 2011;31:363-73.

19. Gomes MVDM, Pelosi GG. Epigenetic vulnerability and the environmental influence on health. Exp Biol Med (Maywood). 2013;238:859-65.

20. Ho S-M, Johnson A, Tarapore P, Janakiram V, Zhang X, Leung Y$\mathrm{K}$. Environmental epigenetics and its implication on disease risk and health outcomes. ILAR J. 2012;53:289-305.

21. Gudsnuk K, Champagne FA. Epigenetic influence of stress and the social environment. ILAR J. 2012;53:279-88.

22. Phillips DM. The presence of acetyl groups of histones. Biochem J. 1963;87:258-63.

23. Gräff J, Tsai L-H. Histone acetylation: molecular mnemonics on the chromatin. Nat Rev Neurosci. 2013;14:97-111.

24. Martin AM, Pouchnik DJ, Walker JL, Wyrick JJ. Redundant roles for histone $\mathrm{H} 3 \mathrm{~N}$-terminal lysine residues in subtelomeric gene 
repression in Saccharomyces cerevisiae. Genetics. 2004;167:112332.

25. He S, Tong Q, Bishop DK, Zhang Y. Histone methyltransferase and histone methylation in inflammatory T-cell responses. Immunotherapy. 2013;5:989-1004.

26. Mosammaparast N, Shi Y. Reversal of histone methylation: biochemical and molecular mechanisms of histone demethylases. Annu Rev Biochem. 2010;79:155-79.

27. Rossetto D, Avvakumov N, Côté J. Histone phosphorylation: a chromatin modification involved in diverse nuclear events. Epigenetics. 2012;7:1098-108.

28. Weake VM, Workman JL. Histone ubiquitination: triggering gene activity. Mol Cell. 2008;29:653-63.

29. Jenuwein T, Allis CD. Translating the histone code. Science. 2001;293:1074-80.

30. Horikoshi M. Histone acetylation: from code to web and router via intrinsically disordered regions. Curr Pharm Des. 2013;19:501942.

31. Dhall A, Chatterjee C. Chemical approaches to understand the language of histone modifications. ACS Chem Biol. 2011;6:987-99.

32. Sahar S, Sassone-Corsi P. The epigenetic language of circadian clocks. Handb Exp Pharmacol. 2013:29-44.

33. Lee J-S, Smith E, Shilatifard A. The language of histone crosstalk. Cell. 2010;142:682-5.

34. Ren B, Robert F, Wyrick JJ, Aparicio O, Jennings EG, Simon I, et al. Genome-wide location and function of DNA binding proteins. Science. 2000;290:2306-9.

35. Iyer VR, Horak CE, Scafe CS, Botstein D, Snyder M, Brown PO. Genomic binding sites of the yeast cell-cycle transcription factors SBF and MBF. Nature. 2001;409:533-8.

36. Bernstein BE, Meissner A, Lander ES. The mammalian epigenome. Cell. 2007;128:669-81.

37. Roh TY, Ngau WC, Cui K, Landsman D, Zhao K. High-resolution genome-wide mapping of histone modifications. Nat Biotechnol. 2004;22:1013-6.

38. Roh TY, Zhao K. High-resolution, genome-wide mapping of chromatin modifications by GMAT. Methods Mol Biol. 2008;387:95-108.

39. Baylin SB, Schuebel KE. Genomic biology: the epigenomic era opens. Nature. 2007;448:548-9.

40. Perissi V, Jepsen K, Glass CK, Rosenfeld MG. Deconstructing repression: evolving models of co-repressor action. Nat Rev Genet. 2010;11: 109-23.

41. Wang Z, Schones DE, Zhao K. Characterization of human epigenomes. Curr Opin Genet Dev. 2009;19:127-34.

42. Rosenfeld JA, Wang Z, Schones DE, Zhao K, DeSalle R, Zhang MQ. Determination of enriched histone modifications in non-genic portions of the human genome. BMC Genomics. 2009;10:143.

43. Mikkelsen TS, Ku M, Jaffe DB, Issac B, Lieberman E, Giannoukos $\mathrm{G}$, et al. Genome-wide maps of chromatin state in pluripotent and lineage-committed cells. Nature. 2007;448:553-60.

44. Liu C, Lu F, Cui X, Cao X. Histone methylation in higher plants. Annu Rev Plant Biol. 2010;61:395-420.

45. Dudakovic A, Evans JM, Li Y, Middha S, McGee-Lawrence ME, van Wijnen AJ, et al. Histone deacetylase inhibition promotes osteoblast maturation by altering the Histone 4 (H4) epigenome and reduces akt phosphorylation. J Biol Chem. 2013.

46. Ferrari R, Su T, Li B, Bonora G, Oberai A, Chan Y, et al. Reorganization of the host epigenome by a viral oncogene. Genome Res. 2012;22:1212-21.

47. Hyllus D, Stein C, Schnabel K, Schiltz E, Imhof A, Dou Y, et al. PRMT6-mediated methylation of R2 in histone $\mathrm{H} 3$ antagonizes $\mathrm{H} 3$ K4 trimethylation. Genes Dev. 2007;21:3369-80.

48. Bernstein BE, Mikkelsen TS, Xie X, Kamal M, Huebert DJ, Cuff J, et al. A bivalent chromatin structure marks key developmental genes in embryonic stem cells. Cell. 2006;125:315-26.
49. Roh TY, Cuddapah S, Cui K, Zhao K. The genomic landscape of histone modifications in human T cells. Proc Natl Acad Sci U S A. 2006;103:15782-7.

50. Core LJ, Waterfall JJ, Lis JT. Nascent RNA sequencing reveals widespread pausing and divergent initiation at human promoters. Science. 2008;322:1845-8.

51. Vastenhouw NL, Schier AF. Bivalent histone modifications in early embryogenesis. Curr Opin Cell Biol. 2012;24:374-86.

52. Jiang H, Shukla A, Wang X, Chen WY, Bernstein BE, Roeder RG. Role for Dpy-30 in ES cell-fate specification by regulation of H3K4 methylation within bivalent domains. Cell. 2011;144:513-25.

53. Kuang FL, Luo Z, Scharff MD. $\mathrm{H} 3$ trimethyl $\mathrm{K} 9$ and $\mathrm{H} 3$ acetyl $\mathrm{K} 9$ chromatin modifications are associated with class switch recombination. Proc Natl Acad Sci U S A. 2009;106:5288-93.

54. Deal RB, Henikoff S. Capturing the dynamic epigenome. Genome Biol. 2010;11:218.

55. Gunesdogan U, Jackle H, Herzig A. A genetic system to assess in vivo the functions of histones and histone modifications in higher eukaryotes. EMBO Rep. 2010;11:772-6.

56. Dai J, Hyland EM, Yuan DS, Huang H, Bader JS, Boeke JD. Probing nucleosome function: a highly versatile library of synthetic histone $\mathrm{H} 3$ and H4 mutants. Cell. 2008;134:1066-78.

57. Suter M, Ma J, Harris A, Patterson L, Brown KA, Shope C, et al. Maternal tobacco use modestly alters correlated epigenome-wide placental DNA methylation and gene expression. Epigenetics. 2011;6:1284-94.

58. Fanelli M, Amatori S, Barozzi I, Minucci S. Chromatin immunoprecipitation and high-throughput sequencing from paraffinembedded pathology tissue. Nat Protoc. 2011;6:1905-19.

59. Zhou X, Li Q, Arita A, Sun H, Costa M. Effects of nickel, chromate, and arsenite on histone 3 lysine methylation. Toxicol Appl Pharmacol. 2009;236:78-84.

60. Jo WJ, Ren X, Chu F, Aleshin M, Wintz H, Burlingame A, et al. Acetylated H4K16 by MYST1 protects UROtsa cells from arsenic toxicity and is decreased following chronic arsenic exposure. Toxicol Appl Pharmacol. 2009;241:294-302.

61. Cronican AA, Fitz NF, Carter A, Saleem M, Shiva S, Barchowsky A, et al. Genome-wide alteration of histone H3K9 acetylation pattern in mouse offspring prenatally exposed to arsenic. PLoS ONE. 2013;8:e53478. This paper is among the first to report ChIP-seq application in histone modifications caused by environmental factors prenatally.

62. Li J, Chen P, Sinogeeva N, Gorospe M, Wersto RP, Chrest FJ, et al. Arsenic trioxide promotes histone $\mathrm{H} 3$ phosphoacetylation at the chromatin of CASPASE-10 in acute promyelocytic leukemia cells. J Biol Chem. 2002;277:49504-10.

63. Lee YW, Klein CB, Kargacin B, Salnikow K, Kitahara J, Dowjat K, et al. Carcinogenic nickel silences gene expression by chromatin condensation and DNA methylation: a new model for epigenetic carcinogens. Mol Cell Biol. 1995; 15:2547-57.

64. Davidson TL, Chen H, Di Toro DM, D'Angelo G, Costa M. Soluble nickel inhibits HIF-prolyl-hydroxylases creating persistent hypoxic signaling in A549 cells. Mol Carcinog. 2006;45:479-89.

65. Arita A, Niu J, Qu Q, Zhao N, Ruan Y, Nadas A, et al. Global levels of histone modifications in peripheral blood mononuclear cells of subjects with exposure to nickel. Environ Health Perspect. 2012;120:198-203.

66. Kang J, Zhang Y, Chen J, Chen H, Lin C, Wang Q, et al. Nickelinduced histone hypoacetylation: the role of reactive oxygen species. Toxicol Sci. 2003;74:279-86.

67. Ke Q, Ellen TP, Costa M. Nickel compounds induce histone ubiquitination by inhibiting histone deubiquitinating enzyme activity. Toxicol Appl Pharmacol. 2008;228:190-9.

68. Tchou-Wong KM, Kiok K, Tang Z, Kluz T, Arita A, Smith PR, et al. Effects of nickel treatment on H3K4 trimethylation and gene expression. PLoS One. 2011;6:e17728. This paper demonstrates how the 
ChIP-seq technique can greatly help researchers understand the particular histone modification patterns caused by environmental factors.

69. Esse R, Florindo C, Imbard A, Rocha MS, de Vriese AS, Smulders $\mathrm{YM}$, et al. Global protein and histone arginine methylation are affected in a tissue-specific manner in a rat model of diet-induced hyperhomocysteinemia. Biochim Biophys Acta. 2013;1832:1708-14.

70. Gopinathan G, Kolokythas A, Luan X, Diekwisch TGH. Epigenetic marks define the lineage and differentiation potential of two distinct neural crest-derived intermediate odontogenic progenitor populations. Stem Cells Dev. 2013;22:1763-78.

71. Zhang Q, Ramlee MK, Brunmeir R, Villanueva CJ, Halperin D, Xu F. Dynamic and distinct histone modifications modulate the expression of key adipogenesis regulatory genes. Cell Cycle. 2012;11: 4310-22.

72. Zou Y, Su Z, Huang W, Gu X. Histone modification pattern evolution after yeast gene duplication. BMC Evol Biol. 2012;12:111.

73. Heimann L, Horst I, Perduns R, Dreesen B, Offermann S, Peterhansel C. A common histone modification code on $\mathrm{C} 4$ genes in maize and its conservation in sorghum and setaria italica. Plant Physiol. 2013;162:456-69.

74. Khan DH, Davie JR. HDAC inhibitors prevent the induction of the immediate-early gene FOSL1, but do not alter the nucleosome response. FEBS Lett. 2013;587:1510-7.

75. Young LC, McDonald DW, Hendzel MJ. Kdm4b histone demethylase is a DNA damage response protein and confers a survival advantage following $\gamma$-irradiation. J Biol Chem. 2013;288:21376-88.

76. Miller AC, Cohen S, Stewart M, Rivas R, Lison P. Radioprotection by the histone deacetylase inhibitor phenylbutyrate. Radiat Environ Biophys. 2011;50:585-96.

77. Groselj B, Sharma NL, Hamdy FC, Kerr M, Kiltie AE. Histone deacetylase inhibitors as radiosensitisers. Effects on DNA damage signalling and repair. Br J Cancer. 2013;108:748-54.

78. Robert C, Rassool FV. HDAC inhibitors: roles of DNA damage and repair. Adv Cancer Res. 2012;116:87-129.

79. Gagnidze K, Weil ZM, Faustino LC, Schaafsma SM, Pfaff DW. Early histone modifications in the ventromedial hypothalamus and preoptic area following oestradiol administration. J Neuroendocrinol. 2013.

80. Shan L, Xing S, Yang H-C, Zhang H, Margolick JB, Siliciano RF. Unique characteristics of histone deacetylase inhibitors in reactivation of latent HIV-1 in Bcl-2-transduced primary resting CD4+ T cells. J Antimicrob Chemother. 2013.

81. Sani E, Herzyk P, Perrella G, Colot V, Amtmann A. Hyperosmotic priming of Arabidopsis seedlings establishes a long-term somatic memory accompanied by specific changes of the epigenome. Genome Biol. 2013;14:R59.

82. Shahhoseini M, Taghizadeh Z, Hatami M, Baharvand H. Retinoic acid dependent histone 3 demethylation of the clustered HOX genes during neural differentiation of human embryonic stem cells. Biochem Cell Biol. 2013;91:116-22.

83. Sharma P, Wollenberg K, Sellers M, Zainabadi K, Galinsky K, Moss E, et al. An epigenetic antimalarial resistance mechanism involving parasite genes linked to nutrient uptake. J Biol Chem. 2013;288:19429-40
84. Ciccarelli A, Calza A, Santoru F, Grasso F, Concas A, SassoèPognetto M, et al. Morphine withdrawal produces ERKdependent and ERK-independent epigenetic marks in neurons of the nucleus accumbens and lateral septum. Neuropharmacology. 2013;70:168-79.

85. Allis CD, Cermakian N, Crosio C, Sassone-Corsi P. Light induces chromatin modification in cells of the mammalian circadian clock. Nat Neurosci. 2000;3:1241+

86. Feng D, Lazar MA. Clocks, metabolism, and the epigenome. Mol Cell. 2012;47:158-67.

87. Malapeira J, Khaitova LC, Mas P. Ordered changes in histone modifications at the core of the Arabidopsis circadian clock. Proc Natl Acad Sci U S A. 2012;109:21540-5.

88. Song H-R, Noh Y-S. Rhythmic oscillation of histone acetylation and methylation at the Arabidopsis central clock loci. Mol Cells. 2012;34:279-87.

89. Masri S, Zocchi L, Katada S, Mora E, Sassone-Corsi P. The circadian clock transcriptional complex: metabolic feedback intersects with epigenetic control. Ann N Y Acad Sci. 2012;1264:103-9.

90. Koike N, Yoo S-H, Huang H-C, Kumar V, Lee C, Kim T-K, et al. Transcriptional architecture and chromatin landscape of the core circadian clock in mammals. Science. 2012;338:349-54.

91. Valekunja UK, Edgar RS, Oklejewicz M, van der Horst GTJ, O'Neill JS, Tamanini F, et al. Histone methyltransferase MLL3 contributes to genome-scale circadian transcription. Proc Natl Acad Sci U S A. 2013;110:1554-9. This recent article provides an example of using the ChIP-seq technique to address genomewide dynamics of histone modification patterns upon periodic changes in environmental factors.

92. Hon GC, Rajagopal N, Shen Y, McCleary DF, Yue F, Dang MD, et al. Epigenetic memory at embryonic enhancers identified in DNA methylation maps from adult mouse tissues. Nat Genet. 2013.

93. Guan Q, Haroon S, Bravo DG, Will JL, Gasch AP. Cellular memory of acquired stress resistance in Saccharomyces cerevisiae. Genetics. 2012;192:495-505.

94. Light WH, Freaney J, Sood V, Thompson A, D'Urso A, Horvath $\mathrm{CM}$, et al. A conserved role for human Nup98 in altering chromatin structure and promoting epigenetic transcriptional memory. PLoS Biol. 2013;11:e1001524.

95. $\mathrm{Ku} \mathrm{CS}$, Naidoo N, Wu M, Soong R. Studying the epigenome using next generation sequencing. J Med Genet. 2011;48:721-30.

96. Mitchell L, Huard S, Cotrut M, Pourhanifeh-Lemeri R, Steunou AL, Hamza A, et al. mChIP-KAT-MS, a method to map protein interactions and acetylation sites for lysine acetyltransferases. Proc Natl Acad Sci U S A. 2013;110:E1641-1650.

97. Chu F, Ren X, Chasse A, Hickman T, Zhang L, Yuh J, et al. Quantitative mass spectrometry reveals the epigenome as a target of arsenic. Chem Biol Interact. 2011;192:113-7.

98. Shi L, Cui S, Engel JD, Tanabe O. Lysine-specific demethylase 1 is a therapeutic target for fetal hemoglobin induction. Nat Med. 2013;19:291-4. This paper presents possible future applications of histone modification patterns.

99. Lelli KM, Slattery M, Mann RS. Disentangling the many layers of eukaryotic transcriptional regulation. Annu Rev Genet. 2012;46: 43-68. 